\title{
Larvicidal and Pupicidal activity of Clerodendrum philippinum Schauer Leaf Extracts against Anopheles stephensi and Aedes aegypti
}

\author{
Pranati Dhal', Jyoti Ranjan Rout ${ }^{2,}$, Preeti Krishna Dash', Sagorika Panda', Pallabi Pati ${ }^{3}$, Chandi Charan Rath ${ }^{4}$, \\ Chinmay Pradhan ${ }^{1}$, Santi Lata Sahoo ${ }^{1}$
}

\section{Pranati Dhal', Jyoti Ranjan Rout ${ }^{2, *}$, Preeti Krishna Dash ${ }^{1}$, Sagorika Panda', Pallabi Pati ${ }^{3}$, Chandi Charan Rath ${ }^{4}$, Chinmay Pradhan', Santi Lata Sahoo'}

\author{
${ }^{1}$ Biochemistry and Molecular Biology \\ Laboratory, Post Graduate Department \\ of Botany, Utkal University, Vani Vihar, \\ Bhubaneswar-751004, Odisha, INDIA. \\ ${ }^{2} \mathrm{~S}$ chool of Biological Sciences, Asian \\ Institute of Public Health, Bhu- \\ baneswar-752101, Odisha, INDIA. \\ ${ }^{3}$ Molecular Epidemiology, ICMR-Region- \\ al Medical Research Centre, Chan- \\ drasekharpur, Bhubaneswar-751023, \\ Odisha, INDIA. \\ ${ }^{4}$ Department of Botany, College of Basic \\ Science and Humanities, Orissa Uni- \\ versity of Agriculture and Technology, \\ Bhubaneswar-751003, Odisha, INDIA. \\ Correspondence \\ Dr. Jyoti Ranjan Rout, PhD, FSPP \\ School of Biological Sciences, \\ Asian Institute of Public Health, \\ Bhubaneswar-752101, Odisha, INDIA \\ Phone no : +91 9438047975 \\ E-mail: routjr@aiph.ac.in \\ History \\ - Submission Date: 23-04-2018 \\ - Review completed: 28-06-2018, \\ - Accepted Date: 23-07-2018
}

\section{DOI : 10.5530/pj.2018.6.194}

\section{Article Available online}

http://www.phcogj.com/v10/i6

\section{Copyright}

(C) 2018 Phcog.Net. This is an openaccess article distributed under the terms of the Creative Commons Attribution 4.0 International license.

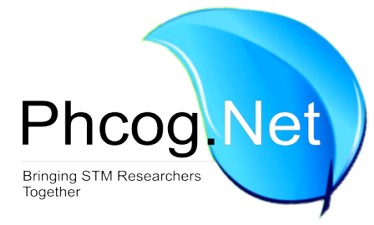

\begin{abstract}
Objective: The purpose of this study is to investigate the larvicidal and pupicidal activity of Clerodendrum philippinum leaf extracts against disease causing vectors Anopheles stephensi and Aedes aegypti. Methods: Five different concentrations (200, 300, 400, 500 and 600 ppm) of leaves were prepared by using aqueous (distilled water), ethanol, methanol, n-hexane, chloroform and tested for both the activity. The $\mathrm{LC}_{50}$ and $\mathrm{LC}_{90}$ values of leaf extracts were determined with the help of probit analysis. Results: Among the different extracts of leaf screened, the ethanol extract of $C$. philippinum was recorded the highest larvicidal and pupicidal activity of $100 \pm 1.9 \%$ ( $1^{\text {st }}$ instar) and $58 \pm 0.8 \%$ at 600 ppm concentration for controlling An. stephensi, respectively. Similarly for Ae. aegypti, $97 \pm 1.2$ ( $1^{\text {st }}$ instar) and $51 \pm 0.5$ (pupa) percentage of inhibition were achieved for the same solvent extracts of the leaf which were maximum than others. Moreover, the values of $\mathrm{LC}_{50}$ and $\mathrm{LC}_{90}$ clearly indicate that the activity of both larvicidal and pupicidal are not only solvent extracts dependant but also depend on their concentration. Conclusion: The obtained data highlight the potential role of ethanolic extracts of $C$. philippinum for controlling An. stephensiand Ae. aegypti mosquitoes at their larval and/ pupal stages of development.

Key words: Larvicidal activity, Pupicidal activity, Clerodendrum philippinum, Anopheles stephensi, Aedes aegypti.
\end{abstract}

\section{INTRODUCTION}

Mosquitoes are serious human disease causing insects which transmit many dreadful diseases and therefore they are considered as 'public enemy number one.'.-2 They are contributing as major public health issues by transmitting many life threatening diseases like malaria, dengue, chikungunya, filariasis, encephalitis, yellow fever and West Nile fever in almost all developed and developing countries of world. ${ }^{3-4}$ Anopheles stephensi is a primary vector for malaria whereas, Aedes aegypti is responsible for causing dengue as well as chikungunya mainly in Asian countries including India. ${ }^{5-6}$ Malaria caused by An. stephensi has become a major contribution in India and are particularly transmitting in the urban and industrial area. ${ }^{7-8}$ In other hands, more than 70,000 and 18,000 cases of dengue and chikungunya, respectively are reported in India caused by Ae. aegypti. ${ }^{9}$

Many control majors (environmental sanitation, epidemiological, surveillance, laboratory and research support and education) are available to check against the vectors, those are responsible for spreading the different life threatening disease. ${ }^{10}$ Moreover, synthetic insecticides are applied to control the agents but have resistant and that might be due to repeated use of such products. It has also caused the environmental threat by destroying non-target organisms and raised adverse effects on both environmental quality and food chain. ${ }^{4,11}$ For which, Integrated Mosquito Management (IMM) emphasizes the application of alternative strategies to control the larva or pupa stages of mosquito. Under such conditions, plants or plant based products showed their effectiveness as controlling agents of mosquitoes which are safer from environment point of view and target specific. They possess rich source of secondary metabolites which having insecticidal activity (antibacterial, antifungal and larvicidal potential) and is possible due to presence of active phenols, alkaloids, terpenoids, coumarins, polysaccharides and flavonoids etc. ${ }^{12-13}$ In fact, many studies have been conducted to find out the effectiveness of different plant extracts against larvicidal and pupicidal activity of mosquito, ${ }^{4,12,14-17}$ but there is no such report against Clerodendrum philippinum.
Cite this article: Dhal P, Rout JR, Dash PK, Panda S, Pati P, Rath CC, Pradhan C, Sahoo SL. Larvicidal and Pupicidal activity of Clerodendrum philippinum Schauer Leaf Extracts against Anopheles stephensi and Aedes aegypti. Pharmacog J. 2018;10(6):1137-42. 
Clerodendrum philippinum Schauer is an important medicinal plant, which belongs to the family Verbenaceae. The plant is also called Peanut Butter plant as the leaf spreads after peeling a peanut butter scent. ${ }^{18-19} \mathrm{It}$ is a semi-woody perennial shrub found in Southern part of Asia and in India, abundantly available in various states like Karnataka, Kerala, Tamil Nadu and Odisha. It grows up to a height of $10 \mathrm{ft}$ tall. Leaves are broad, up to $1 \mathrm{ft} \mathrm{cm}$ long and wide, margins toothed, somewhat lobed. Flowers are found in tight clusters, white with pink or red tinge and highly fragranced. ${ }^{20-21}$ Different parts of the plant have been used against various ailments like inflammatory disorders, cancer, diabetic, rheumatism, asthma, constipation, colic pain, jaundice, syphilis, typhoid etc. ${ }^{18,20,22-23}$ It is also reported that the plant has antibacterial and antifungal properties, however no evidence is found regarding its antimalarial/ larvicidal activity. ${ }^{24-25}$ Hence, the present study is undertaken to evaluate the larvicidal and pupicidal activity of leaf extracts as well as flower essential oil against two vectors, Anopheles stephensi and Aedes aegypti.

\section{MATERIALS AND METHODS}

\section{Collection and preparation of leaf extracts}

Healthy leaves of C. philippinum were collected from the botanical garden of Post Graduate Department of Botany, Utkal University and were washed and air dried under the environmental temperature of $27^{\circ} \mathrm{C}-37^{\circ} \mathrm{C}$. The dried leaves were powdered by using a mortar and pestle. The powdered sample was eluted using a Soxhlet apparatus with different solvents [aqueous, ethanol, methanol, n-hexane, chloroform) sequentially in a ratio of $1: 10$ for a period of $72 \mathrm{~h}$ each and filtered. The diluted extracts were concentrated $(200,300,400,500$ and $600 \mathrm{ppm})$ at low temperature using a rotary evaporator and stored at $-4^{\circ} \mathrm{C}$ for further analysis.

\section{Collection of eggs and larval culture}

The eggs of An. stephensi and Ae. aegypti were provided by Department of Entomology, Regional Medical Research Centre, Bhubaneswar, Odisha, India. The eggs were brought to the laboratory and kept in plastic and enamel tray containing normal tap water for hatching. The larvae were fed on a diet having fine a mixture of dog biscuits and dry yeast in a ratio of $3: 1$, till the pupal stage.

\section{Maintenance of pupae and adults}

Developed pupae were relocated in to separate plastic cup containing tap water and were kept under periodical check-up for emerging into adults. Larvae/ pupae were maintained throughout at $27 \pm 2^{\circ} \mathrm{C}$ and $75-85 \%$ of relative humidity with a photoperiod of $14 \mathrm{~h} / 10 \mathrm{~h}$ light and dark cycles. After pupation, they were allowed inside a mosquito cage for becoming adults. Prior to blood feeding, $10 \%$ sucrose solution was provided for 3 days and finally, adult female mosquitoes were maintained by providing a blood meal from rabbit (exposed in dorsal side).

\section{Larvicidal and pupicidal activity test}

The bioassay was done as per the standard method of World Health Organization by taking different solvent extracts of leaf. ${ }^{26}$ Five different concentrations $(200,300,400,500$ and $600 \mathrm{ppm})$ of each extract were tested against freshly moulted first $\left(1^{\text {st }}\right)$, second $\left(2^{\text {nd }}\right)$, third $\left(3^{\text {rd }}\right)$ and fourth $\left(4^{\text {th }}\right)$ instar larvae and pupae of An. stephensi and Ae. aegypti. Twenty numbers of first to fourth instar larvae and pupae were introduced into $500 \mathrm{ml}$ thermocol glass containing $249 \mathrm{ml}$ dechlorinated water and $1 \mathrm{ml}$ of desired concentrations of plant extract (dilution was done by dechlorinated water). At each tested concentration, three trials were made and each trial consisted of five replicates. The control was set up by providing $250 \mathrm{ml}$ of dechlorinated water however the larval food was added to all tested and control cups. The larval mortality was observed and recorded after $24 \mathrm{~h}$ of post treatment. The mortalities were recorded by using Abbott's formula (Abbott, 1925).

\section{Statistical analysis}

Results of larval and pupal mortality were the mean of three independent experimental replicates $(n=5)$ and were subjected to probit analysis ${ }^{27}$ for calculating $\mathrm{LC}_{50}, \mathrm{LC}_{90}$ and other statistics at $95 \%$ fiducial limits of upper fiducial limit (UFL) and lower fiducial limit (LFL) and chi-square values were calculated using the SPSS 16.0 version (software package). Values with $P<0.05$ were considered to be statistically significant.

\section{RESULTS}

\section{Larvicidal and pupicidal activity against An. stephensi}

Larvicidal and pupicidal mortality responses from various leaf extracts (aqueous (distilled water), ethanol, methanol, n-hexane, chloroform) of C. philippinum, against malaria causing vector An. stephensi are presented in Tables 1-5. The different concentrations (200, 300, 400, 500 and $600 \mathrm{ppm}$ ) of each extract were tested against $1^{\text {st }}, 2^{\text {nd }}, 3^{\text {rd }}, 4^{\text {th }}$ instar and pupal stages of An. stephensi. Increasing trends of mortality were found against both larval and pupal stages of An. stephensi with raising concentrations (200 to $600 \mathrm{ppm}$ ) of each leaf extracts. A significant mortality was noticed in all applied solvent systems however, best mortality was observed in ethanolic extracts. At $600 \mathrm{ppm}$, the ethanolic extract of leaf exhibited $100 \%$ of results in $1^{\text {st }}$ instar stage of larva and subsequently the percentage of mortality was decreased to $97 \pm 0.8 \%, 85 \pm 0.7 \%$, $73 \pm 0.9 \%\left(2^{\text {nd }}, 3^{\text {rd }}\right.$ and $4^{\text {th }}$ instar, respectively $)$. A similar trend has been noticed for the pupal stage that the maximum mortality (58 $\pm 0.8 \%)$ was achieved in $600 \mathrm{ppm}$ of etanolic extract (Table 2). Furthermore, the $\mathrm{LC}_{50}$ and $\mathrm{LC}_{90}$ values for ethanol extract of $1^{\text {st }}$ instar and pupa were found to be $257.14,811.11$ and $582.04,1185.71 \mathrm{ppm}$ respectively with the chi square values of 1.92 and 0.85 . The values of $\mathrm{LC}_{50}$ and $\mathrm{LC}_{90}$ of other extracts against instars and pupae are shown in Tables 1-5, where the chi square values were statistically significant at $P<0.05$ level.

\section{Larvicidal and pupicidal activity against Ae. aegypti}

The same set up was tried against dengue and chikungunya causing mosquito, Ae aegypti and the results of larvicidal and pupicidal activity of different leaf extracts of C. philippinum were tabulated (Tables 6-10). Similar findings were detected as occurred in An. stephensi that the ethanol extract of leaves gave maximum mortality in comparison to others. $97 \pm 1.2$ and $51 \pm 0.5 \%$ of larval and pupal mortality, respectively were recorded at $600 \mathrm{ppm}$ of ethanol extract, which was maximum to others (Table 6). When the values of larvicidal activity were noticed in all solvent extracts, the effective results were obtained in $1^{\text {st }}$ instar and then followed by $2^{\text {nd }}, 3^{\text {rd }}$ and $4^{\text {th }}$ against individual concentration. Moreover from the solvent efficacy point of view, the hierarchy of mortality (both larval and pupal) was found to be ethanol $>$ methanol $>$ chloroform $>$ n-hexane $>$ aqueous. The $\mathrm{LC}_{50}$ and $\mathrm{LC}_{90}$ values for different extracts against larval and pupal mortality of An. stephensi are represented in Tables 6-10. The tables indicating chi square values were significant at $P<0.05$ (Tables 6-10)

\section{DISCUSSION}

In the present study, probit analysis reveals that $\mathrm{LC}_{50}$ and $\mathrm{LC}_{90}$ of leaf extracts of $C$. philippinum were effective against An. stephensi and Ae. aegypti during the larval and pupal stage. In general, the mortality rate from $1^{\text {st }}$ to $4^{\text {th }}$ instar were cumulatively decreasing which indicate that the extracts created a more toxic environment during the very beginning stages of mosquitoes (that have tried). Leaf extracts produced enhanced mortality with increasing concentrations of extracts and further, it has been noticed that ethanol extracts possessed strong activity than others 
Table 1: Larval and pupal toxicity effect of aqueous extracts of $C$. philippinum against An. stephensi.

\begin{tabular}{|c|c|c|c|c|c|c|c|c|c|c|}
\hline \multirow{2}{*}{$\begin{array}{l}\text { Mosquito } \\
\text { life stage }\end{array}$} & \multicolumn{5}{|c|}{$\%$ of larval and pupal mortality \pm SD } & \multirow[t]{2}{*}{$\mathrm{LC}_{50}$} & \multirow[t]{2}{*}{$\mathrm{LC}_{90}$} & \multicolumn{2}{|c|}{$95 \%$ confidence limit (LFL-UFL) } & \multirow[t]{2}{*}{$X^{2}(d f=4)$} \\
\hline & 200 ppm & 300 ppm & 400 ppm & 500 ppm & 600 ppm & & & $\mathrm{LC}_{50}$ & $\mathrm{LC}_{90}$ & \\
\hline $1^{\text {st }}$ instar & $32 \pm 0.8$ & $42 \pm 0.9$ & $53 \pm 0.9$ & $74 \pm 2.1$ & $85 \pm 1.2$ & 372.72 & 662.50 & $284.72-460.72$ & $622.50-702.50$ & $1.74^{*}$ \\
\hline $2^{\text {nd }}$ instar & $30 \pm 0.9$ & $33 \pm 2.1$ & $46 \pm 1.4$ & $62 \pm 0.9$ & $81 \pm 0.8$ & 431.25 & 799.99 & $210.25-652.25$ & $763.99-835.99$ & $1.70^{*}$ \\
\hline $3^{\text {rd }}$ instar & $27 \pm 0.7$ & $27 \pm 0.9$ & $44 \pm 1.1$ & $56 \pm 0.5$ & $68 \pm 0.5$ & 441.66 & 866.66 & $369.66-513.60$ & $830.66-902.66$ & $0.70^{*}$ \\
\hline $4^{\text {th }}$ instar & $23 \pm 1.9$ & $28 \pm 0.7$ & $38 \pm 0.8$ & $52 \pm 0.8$ & $59 \pm 0.5$ & 485.71 & 1075.02 & $317.71-653.71$ & $969.87-1145.09$ & $0.25^{*}$ \\
\hline Pupa & $10 \pm 1.5$ & $18 \pm 1.9$ & $28 \pm 0.9$ & $33 \pm 0.4$ & $47 \pm 0.8$ & 635.71 & 1187.50 & $537.71-721.04$ & $1067.50-1307.50$ & $0.74^{*}$ \\
\hline
\end{tabular}

Control: Nil mortality; LFL: Lower fiducial limit; (UFL): Upper fiducial limit; $\chi 2$ : Chi square value; $\mathrm{d} f$ : Degrees of freedom; ${ }^{*}$ Significant at $\mathrm{P}<0.05$ level.

Table 2: Larval and pupal toxicity effect of ethanol extracts of $C$. philippinum against An. stephensi.

\begin{tabular}{|c|c|c|c|c|c|c|c|c|c|c|}
\hline \multirow{2}{*}{$\begin{array}{l}\text { Mosquito } \\
\text { life stage }\end{array}$} & \multicolumn{5}{|c|}{$\%$ of larval and pupal mortality \pm SD } & \multirow[t]{2}{*}{$\mathrm{LC}_{50}$} & \multirow[t]{2}{*}{$\mathrm{LC}_{90}$} & \multicolumn{2}{|c|}{$95 \%$ confidence limit (LFL-UFL) } & \multirow[t]{2}{*}{$X^{2}(d f=4)$} \\
\hline & 200 ppm & 300 ppm & 400 ppm & 500 ppm & 600 ppm & & & $\mathrm{LC}_{50}$ & $\mathrm{LC}_{90}$ & \\
\hline $1^{\text {st }}$ instar & $46 \pm 1.1$ & $53 \pm 0.9$ & $59 \pm 0.5$ & $84 \pm 0.7$ & $100 \pm 1.9$ & 257.14 & 811.11 & $229.14-285.14$ & 711.11-911.11 & $1.92^{*}$ \\
\hline $2^{\text {nd }}$ instar & $40 \pm 0.9$ & $43 \pm 0.6$ & $58 \pm 0.8$ & $73 \pm 0.8$ & $97 \pm 0.8$ & 346.66 & 833.33 & $241.66-451.66$ & $737.33-929.33$ & $1.80^{*}$ \\
\hline $3^{\text {rd }}$ instar & $36 \pm 1.2$ & $36 \pm 0.9$ & $51 \pm 0.7$ & $64 \pm 1.1$ & $85 \pm 0.7$ & 393.33 & 953.84 & $183.33-603.33$ & 953.84-1033.84 & $0.90^{*}$ \\
\hline $4^{\text {th }}$ instar & $29 \pm 1.0$ & $30 \pm 1.1$ & $48 \pm 0.6$ & $59 \pm 0.8$ & $73 \pm 0.9$ & 445.45 & 1012.50 & $423.45-467.45$ & $984.50-1040.50$ & $0.31^{*}$ \\
\hline Pupa & $21 \pm 0.7$ & $28 \pm 0.9$ & $34 \pm 0.7$ & $41 \pm 0.6$ & $58 \pm 0.8$ & 582.04 & 1185.71 & $467.41-682.41$ & $1133.71-1237.71$ & $0.85^{*}$ \\
\hline
\end{tabular}

Control: Nil mortality; LFL: Lower fiducial limit; (UFL): Upper fiducial limit; $\chi 2$ : Chi square value; $\mathrm{d} f$ : Degrees of freedom; ${ }^{*}$ : Significant at $\mathrm{P}<0.05$ level

Table 3: Larval and pupal toxicity effect of methanol extracts of $C$. philippinum against An. stephensi.

\begin{tabular}{|c|c|c|c|c|c|c|c|c|c|c|}
\hline \multirow{2}{*}{$\begin{array}{l}\text { Mosquito } \\
\text { life stage }\end{array}$} & \multicolumn{5}{|c|}{$\%$ of larval and pupal mortality \pm SD } & \multirow[t]{2}{*}{$\mathrm{LC}_{50}$} & \multirow[t]{2}{*}{$\mathrm{LC}_{90}$} & \multicolumn{2}{|c|}{$95 \%$ confidence limit (LFL-UFL) } & \multirow[t]{2}{*}{$X^{2}(d f=4)$} \\
\hline & 200 ppm & $300 \mathrm{ppm}$ & $400 \mathrm{ppm}$ & $500 \mathrm{ppm}$ & 600 ppm & & & $\mathrm{LC}_{50}$ & $\mathrm{LC}_{90}$ & \\
\hline $1^{\text {st }}$ instar & $41 \pm 1.3$ & $48 \pm 0.4$ & $57 \pm 0.7$ & $79 \pm 1.9$ & $92 \pm 0.9$ & 355.55 & 625.07 & $292.55-418.55$ & $549.55-701.55$ & $1.89^{*}$ \\
\hline $2^{\text {nd }}$ instar & $38 \pm 0.7$ & $39 \pm 0.3$ & $54 \pm 1.1$ & $68 \pm 0.6$ & $87 \pm 0.4$ & 435.71 & 777.79 & $270.71-600.71$ & $701.79-853.79$ & $1.76^{*}$ \\
\hline $3^{\text {rd }}$ instar & $31 \pm 0.9$ & $31 \pm 0.7$ & $48 \pm 1.5$ & $60 \pm 0.9$ & $78 \pm 0.4$ & 441.66 & 877.77 & $417.66-465.66$ & $841.77-913.77$ & $0.83^{*}$ \\
\hline $4^{\text {th }}$ instar & $27 \pm 1.2$ & $27 \pm 0.9$ & $44 \pm 0.9$ & $56 \pm 0.7$ & $67 \pm 0.3$ & 545.55 & 975.43 & $473.55-617.55$ & $927.05-1023.15$ & $0.27^{\star}$ \\
\hline Pupa & $17 \pm 0.5$ & $24 \pm 0.6$ & $38 \pm 0.6$ & $37 \pm 0.9$ & $55 \pm 0.3$ & 627.77 & 1020.23 & $393.77-861.77$ & $980.20-1060.01$ & $0.81^{*}$ \\
\hline
\end{tabular}

Control: Nil mortality; LFL: Lower fiducial limit; (UFL): Upper fiducial limit; $\chi 2$ : Chi square value; $\mathrm{d} f$ : Degrees of freedom; *: Significant at P<0.05 level.

Table 4: Larval and pupal toxicity effect of $n$-hexane extracts of $C$. philippinum against An. stephensi.

\begin{tabular}{|c|c|c|c|c|c|c|c|c|c|c|}
\hline \multirow{2}{*}{$\begin{array}{l}\text { Mosquito } \\
\text { life stage }\end{array}$} & \multicolumn{5}{|c|}{$\%$ of larval and pupal mortality \pm SD } & \multirow[t]{2}{*}{$\mathrm{LC}_{50}$} & \multirow[t]{2}{*}{$\mathrm{LC}_{90}$} & \multicolumn{2}{|c|}{$95 \%$ confidence limit (LFL-UFL) } & \multirow[t]{2}{*}{$X^{2}(d f=4)$} \\
\hline & 200 ppm & 300 ppm & 400 ppm & 500 ppm & 600 ppm & & & $\mathrm{LC}_{50}$ & $\mathrm{LC}_{90}$ & \\
\hline $1^{\text {st }}$ instar & $35 \pm 0.9$ & $44 \pm 1.5$ & $54 \pm 0.8$ & $75 \pm 1.1$ & $86 \pm 0.8$ & 360.00 & 628.25 & $300.03-420.01$ & $584.25-672.25$ & $1.80^{*}$ \\
\hline $2^{\text {nd }}$ instar & $31 \pm 1.1$ & $35 \pm 1.0$ & $49 \pm 1.9$ & $64 \pm 1.9$ & $82 \pm 0.4$ & 407.33 & 766.66 & $323.33-543.33$ & $718.66-814.66$ & $1.69^{*}$ \\
\hline $3^{\text {rd }}$ instar & $28 \pm 0.9$ & $27 \pm 0.7$ & $45 \pm 0.7$ & $57 \pm 0.7$ & $69 \pm 0.8$ & 441.66 & 800.33 & $381.66-501.66$ & $760.23-840.01$ & $0.75^{*}$ \\
\hline $4^{\text {th }}$ instar & $24 \pm 0.7$ & $23 \pm 0.9$ & $39 \pm 0.1$ & $53 \pm 0.4$ & $61 \pm 0.9$ & 478.57 & 900.00 & $394.57-632.57$ & $860.01-940.43$ & $0.27^{*}$ \\
\hline Pupa & $11 \pm 0.5$ & $21 \pm 0.9$ & $30 \pm 0.6$ & $31 \pm 0.1$ & $49 \pm 1.1$ & 627.77 & 1031.25 & $495.77-769.27$ & $971.25-1091.25$ & $0.78^{*}$ \\
\hline
\end{tabular}

Control: Nil mortality; LFL: Lower fiducial limit; (UFL): Upper fiducial limit; $\chi 2$ : Chi square value; $\mathrm{d} f$ : Degrees of freedom; *: Significant at P $<0.05$ level.

Table 5: Larval and pupal toxicity effect of chloroform extracts of $C$. philippinum against An stephensi.

\begin{tabular}{|c|c|c|c|c|c|c|c|c|c|c|}
\hline \multirow{2}{*}{$\begin{array}{l}\text { Mosquito } \\
\text { life stage }\end{array}$} & \multicolumn{5}{|c|}{$\%$ of larval and pupal mortality \pm SD } & \multirow{2}{*}{$\mathrm{LC}_{50}$} & \multirow[t]{2}{*}{$\mathrm{LC}_{90}$} & \multicolumn{2}{|c|}{$95 \%$ confidence limit (LFL-UFL) } & \multirow[t]{2}{*}{$X^{2}(d f=4)$} \\
\hline & 200 ppm & 300 ppm & 400 ppm & 500 ppm & 600 ppm & & & $\mathrm{LC}_{50}$ & $\mathrm{LC}_{90}$ & \\
\hline $1^{\text {stinstar }}$ & $37 \pm 1.1$ & $46 \pm 0.5$ & $56 \pm 0.6$ & $76 \pm 1.0$ & $88 \pm 1.0$ & 350.00 & 689.04 & $310-390$ & $578.03-765.89$ & $1.85^{*}$ \\
\hline $2^{\text {nd }}$ instar & $33 \pm 0.9$ & $37 \pm 0.9$ & $51 \pm 1.2$ & $65 \pm 1.2$ & $85 \pm 0.3$ & 392.85 & 842.5 & $346.85-494.85$ & $806.50-878.50$ & $1.72^{*}$ \\
\hline $3^{\text {rd }}$ instar & $29 \pm 0.7$ & $28 \pm 0.3$ & $46 \pm 1.0$ & $58 \pm 0.4$ & $70 \pm 0.7$ & 441.66 & 883.65 & $409.66-473.66$ & $799.65-967.65$ & $0.80^{*}$ \\
\hline $4^{\text {th }}$ instar & $25 \pm 0.5$ & $24 \pm 0.5$ & $41 \pm 0.4$ & $54 \pm 0.5$ & $63 \pm 0.5$ & 469.23 & 986.34 & $352.23-586.23$ & $908.23-1145.89$ & $0.24^{*}$ \\
\hline Pupa & $13 \pm 0.9$ & $22 \pm 0.2$ & $32 \pm 0.3$ & $33 \pm 0.6$ & $50 \pm 0.7$ & 600.00 & 1070.25 & $583.00-617.00$ & $1015.23-1198.32$ & $0.77^{*}$ \\
\hline
\end{tabular}

Control: Nil mortality; LFL: Lower fiducial limit; (UFL): Upper fiducial limit; $\chi 2$ : Chi square value; $\mathrm{d} f$ : Degrees of freedom; ${ }^{*}$ : Significant at $\mathrm{P}<0.05$ level. 
Table 6: Larval and pupal toxicity effect of aqueous extracts of C. philippinum against Ae. aegypti.

\begin{tabular}{|c|c|c|c|c|c|c|c|c|c|c|}
\hline \multirow{2}{*}{$\begin{array}{l}\text { Mosquito } \\
\text { life stage }\end{array}$} & \multicolumn{5}{|c|}{$\%$ of larval and pupal mortality \pm SD } & \multirow[t]{2}{*}{$\mathrm{LC}_{50}$} & \multirow[t]{2}{*}{$\mathrm{LC}_{90}$} & \multicolumn{2}{|c|}{$95 \%$ confidence limit (LFL-UFL) } & \multirow[t]{2}{*}{$X^{2}(d f=4)$} \\
\hline & 200 ppm & 300 ppm & 400 ppm & $500 \mathrm{ppm}$ & 600 ppm & & & $\mathrm{LC}_{50}$ & $\mathrm{LC}_{90}$ & \\
\hline $1^{\text {stinstar }}$ & $27 \pm 0.9$ & $31 \pm 0.9$ & $43 \pm 0.8$ & $58 \pm 3.1$ & $81 \pm 0.5$ & 433.33 & 768.45 & $328.33-583.33$ & $695.32-840.56$ & $1.70^{*}$ \\
\hline $2^{\text {nd }}$ instar & $22 \pm 0.4$ & $29 \pm 0.5$ & $34 \pm 2.1$ & $51 \pm 1.1$ & $73 \pm 0.5$ & 494.11 & 850 & $386.78-566.11$ & $814.04-886.53$ & $1.67^{*}$ \\
\hline $3^{\text {rd }}$ instar & $19 \pm 1.6$ & $22 \pm 1.1$ & $32 \pm 0.8$ & $43 \pm 0.6$ & $66 \pm 0.3$ & 521.73 & 900.68 & $444.73-598.73$ & $825.13-975.98$ & $0.68^{*}$ \\
\hline $4^{\text {th }}$ instar & $11 \pm 0.6$ & $18 \pm 0.5$ & $26 \pm 0.3$ & $41 \pm 1.1$ & $53 \pm 1.2$ & 575.03 & 1085.35 & $479.00-671.04$ & $925.00-1234.09$ & $0.19^{*}$ \\
\hline Pupa & $7 \pm 0.2$ & $11 \pm 0.7$ & $22 \pm 0.4$ & $30 \pm 2.2$ & $41 \pm 0.9$ & 645.45 & 1133.33 & $546.45-744.45$ & $997.33-1269.33$ & $0.61^{*}$ \\
\hline
\end{tabular}

Control: Nil mortality; LFL: Lower fiducial limit; (UFL): Upper fiducial limit; $\chi 2$ : Chi square value; $\mathrm{d} f$ : Degrees of freedom; ${ }^{*}$ : Significant at $\mathrm{P}<0.05$ level.

Table 7: Larval and pupal toxicity effect of ethanol extracts of $C$. philippinum against Ae. aegypti.

\begin{tabular}{|c|c|c|c|c|c|c|c|c|c|c|}
\hline \multirow{2}{*}{$\begin{array}{l}\text { Mosquito } \\
\text { life stage }\end{array}$} & \multicolumn{5}{|c|}{$\%$ of larval and pupal mortality \pm SD } & \multirow[t]{2}{*}{$L C_{50}$} & \multirow[t]{2}{*}{$L C_{90}$} & \multicolumn{2}{|c|}{$95 \%$ confidence limit (LFL-UFL) } & \multirow[t]{2}{*}{$X^{2}(d f=4)$} \\
\hline & 200 ppm & 300 ppm & 400 ppm & 500 ppm & 600 ppm & & & $\mathrm{LC}_{50}$ & $\mathrm{LC}_{90}$ & \\
\hline $1^{\text {st }}$ instar & $35 \pm 0.7$ & $42 \pm 0.9$ & $51 \pm 0.9$ & $69 \pm 0.6$ & $97 \pm 1.2$ & 388.88 & 700.11 & $294.88-460.88$ & $564.12-836.09$ & $1.87^{*}$ \\
\hline $2^{\text {nd }}$ instar & $31 \pm 0.6$ & $38 \pm 0.2$ & $46 \pm 0.5$ & $62 \pm 0.7$ & $92 \pm 0.6$ & 431.25 & 733.33 & $399.25-463.25$ & $697.33-769.33$ & $1.76^{*}$ \\
\hline $3^{\text {rd }}$ instar & $27 \pm 0.3$ & $28 \pm 0.7$ & $41 \pm 0.2$ & $52 \pm 0.4$ & $87 \pm 0.5$ & 481.81 & 768.75 & $382.81-580.81$ & $628.75-908.75$ & $0.85^{*}$ \\
\hline $4^{\text {th }}$ instar & $21 \pm 0.9$ & $26 \pm 0.9$ & $37 \pm 0.7$ & $48 \pm 0.9$ & $65 \pm 0.4$ & 529.41 & 883.65 & 507.41-551.41 & $847.65-919.65$ & $0.27^{*}$ \\
\hline Pupa & $17 \pm 0.19$ & $22 \pm 0.8$ & $32 \pm 0.9$ & $39 \pm 0.6$ & $51 \pm 0.5$ & 591.66 & 1183.33 & $519.66-673.66$ & $1147.33-1219.33$ & $0.73^{*}$ \\
\hline
\end{tabular}

Control: Nil mortality; LFL: Lower fiducial limit; (UFL): Upper fiducial limit; $\chi 2$ : Chi square value; $\mathrm{d} f$ : Degrees of freedom; ${ }^{*}$ : Significant at $\mathrm{P}<0.05$ level.

Table 8: Larval and pupal toxicity effect of methanol extracts of C. philippinum against Ae. aegypti.

\begin{tabular}{|c|c|c|c|c|c|c|c|c|c|c|}
\hline \multirow{2}{*}{$\begin{array}{l}\text { Mosquito } \\
\text { life stage }\end{array}$} & \multicolumn{5}{|c|}{$\%$ of larval and pupal mortality \pm SD } & \multirow[t]{2}{*}{$\mathrm{LC}_{50}$} & \multirow[t]{2}{*}{$\mathrm{LC}_{90}$} & \multicolumn{2}{|c|}{$95 \%$ confidence limit (LFL-UFL) } & \multirow[t]{2}{*}{$X^{2}(d f=4)$} \\
\hline & 200 ppm & 300 ppm & $400 \mathrm{ppm}$ & 500 ppm & 600 ppm & & & $\mathrm{LC}_{50}$ & $\mathrm{LC}_{90}$ & \\
\hline $1^{\text {st }}$ instar & $33 \pm 0.6$ & $38 \pm 1.9$ & $49 \pm 0.8$ & $67 \pm 0.4$ & $86 \pm 1.4$ & 404.90 & 618.57 & $397.02-428.77$ & $547.57-698.57$ & $1.85^{*}$ \\
\hline $2^{\text {nd }}$ instar & $29 \pm 0.8$ & $33 \pm 0.8$ & $44 \pm 0.9$ & $57 \pm 1.2$ & $78 \pm 0.8$ & 538.46 & 663.63 & $460.46-616.46$ & $563.63-763.63$ & $1.71^{*}$ \\
\hline $3^{\text {rd }}$ instar & $25 \pm 0.9$ & $28 \pm 0.4$ & $39 \pm 1.2$ & $49 \pm 0.6$ & $70 \pm 0.6$ & 510.01 & 790.64 & 500.09-520.09 & $746.64-834.64$ & $0.79^{*}$ \\
\hline $4^{\text {th }}$ instar & $19 \pm 0.4$ & $23 \pm 0.6$ & $35 \pm 0.5$ & $46 \pm 0.4$ & $60 \pm 0.4$ & 536.66 & 868.66 & 492.66-580.66 & $812.66-921.66$ & $0.24^{*}$ \\
\hline Pupa & $14 \pm 0.2$ & $19 \pm 0.2$ & $28 \pm 0.6$ & $36 \pm 0.5$ & $46 \pm 0.8$ & 650.02 & 979.32 & 610.11-690.09 & $861.32-1097.35$ & $0.70^{*}$ \\
\hline
\end{tabular}

Control: Nil mortality; LFL: Lower fiducial limit; (UFL): Upper fiducial limit; $\chi 2$ : Chi square value; $\mathrm{d} f$ : Degrees of freedom; ${ }^{*}$ : Significant at $\mathrm{P}<0.05$ level.

Table 9: Larval and pupal toxicity effect of $\mathrm{n}$-hexane extracts of $C$. philippinum against Ae. aegypti.

\begin{tabular}{|c|c|c|c|c|c|c|c|c|c|c|}
\hline \multirow{2}{*}{$\begin{array}{l}\text { Mosquito } \\
\text { life stage }\end{array}$} & \multicolumn{5}{|c|}{$\%$ of larval and pupal mortality \pm SD } & \multirow[t]{2}{*}{$\mathrm{LC}_{50}$} & \multirow[t]{2}{*}{$\mathrm{LC}_{90}$} & \multicolumn{2}{|c|}{$95 \%$ confidence limit (LFL-UFL) } & \multirow[t]{2}{*}{$X^{2}(d f=4)$} \\
\hline & 200 ppm & 300 ppm & 400 ppm & 500 ppm & 600 ppm & & & $\mathrm{LC}_{50}$ & $\mathrm{LC}_{90}$ & \\
\hline $1^{\text {st }}$ instar & $29 \pm 1.0$ & $33 \pm 1.3$ & $45 \pm 1.2$ & $61 \pm 1.1$ & $83 \pm 0.9$ & 431.25 & 663.63 & $371.25-491.25$ & $619.75-707.75$ & $1.72^{*}$ \\
\hline $2^{\text {nd }}$ instar & $24 \pm 0.7$ & $30 \pm 0.1$ & $37 \pm 0.9$ & $53 \pm 1.0$ & $74 \pm 0.3$ & 481.25 & 812.50 & $501.25-689.25$ & $713.50-772.50$ & $1.61^{*}$ \\
\hline $3^{\text {rd }}$ instar & $21 \pm 0.4$ & $24 \pm 0.9$ & $34 \pm 0.3$ & $45 \pm 0.6$ & $67 \pm 0.4$ & 525.45 & 842.85 & $464.05-618.95$ & $814.85-870.85$ & $0.65^{*}$ \\
\hline $4^{\text {th }}$ instar & $15 \pm 0.2$ & $19 \pm 0.2$ & $29 \pm 0.7$ & $42 \pm 0.4$ & $55 \pm 0.9$ & 561.53 & 953.84 & $457.53-665.53$ & $897.46-1125.07$ & $0.17^{*}$ \\
\hline Pupa & $8 \pm 0.6$ & $13 \pm 0.21$ & $24 \pm 1.1$ & $28 \pm 0.9$ & $43 \pm 0.6$ & 620.33 & 1068.76 & $503.33-701.33$ & $1004.76-1132.76$ & $0.68^{*}$ \\
\hline
\end{tabular}

Control: Nil mortality; LFL: Lower fiducial limit; (UFL): Upper fiducial limit; $\chi 2$ : Chi square value; $\mathrm{d} f$ : Degrees of freedom; ${ }^{*}$ Significant at $\mathrm{P}<0.05$ level.

Table 10: Larval and pupal toxicity effect of chloroform extracts of C. philippinum against Ae. aegypti.

\begin{tabular}{|c|c|c|c|c|c|c|c|c|c|c|}
\hline \multirow{2}{*}{$\begin{array}{l}\text { Mosquito } \\
\text { life stage }\end{array}$} & \multicolumn{5}{|c|}{$\%$ of larval and pupal mortality \pm SD } & \multirow[t]{2}{*}{$\mathrm{LC}_{50}$} & \multirow[t]{2}{*}{$\mathrm{LC}_{90}$} & \multicolumn{2}{|c|}{$95 \%$ confidence limit (LFL-UFL) } & \multirow[t]{2}{*}{$X^{2}(d f=4)$} \\
\hline & 200 ppm & 300 ppm & 400 ppm & 500 ppm & 600 ppm & & & $\mathrm{LC}_{50}$ & $\mathrm{LC}_{90}$ & \\
\hline $1^{\text {st }}$ instar & $31 \pm 0.8$ & $35 \pm 1.1$ & $47 \pm 0.6$ & $65 \pm 0.9$ & $84 \pm 2.1$ & 427.27 & 637.65 & $391.27-463.27$ & $561.65-713.65$ & $1.76^{*}$ \\
\hline $2^{\text {nd }}$ instar & $27 \pm 0.5$ & $32 \pm 1.0$ & $39 \pm 0.5$ & $54 \pm 1.9$ & $76 \pm 1.3$ & 476.13 & 658.33 & $428.33-558.33$ & $570.33-746.33$ & $1.64^{*}$ \\
\hline $3^{\text {rd }}$ instar & $23 \pm 0.2$ & $26 \pm 0.7$ & $36 \pm 0.9$ & $47 \pm 0.9$ & $68 \pm 1.2$ & 515.80 & 735.75 & $484.80-546.17$ & $672.80-834.11$ & $0.70^{*}$ \\
\hline $4^{\text {th }}$ instar & $17 \pm 0.7$ & $21 \pm 0.5$ & $31 \pm 0.9$ & $44 \pm 0.9$ & $57 \pm 0.5$ & 546.15 & 876.74 & $468.15-624.15$ & $826.46-928.46$ & $0.19^{*}$ \\
\hline Pupa & $10 \pm 0.5$ & $15 \pm 0.6$ & $26 \pm 0.2$ & $35 \pm 1.5$ & $44 \pm 0.2$ & 655.55 & 982.14 & $601.55-709.55$ & $943.14-1018.14$ & $0.65^{*}$ \\
\hline
\end{tabular}

Control: Nil mortality; LFL: Lower fiducial limit; (UFL): Upper fiducial limit; $\chi 2$ : Chi square value; $\mathrm{d} f$ : Degrees of freedom; ${ }^{\star}$ : Significant at $\mathrm{P}<0.05$ level. 
(Tables 1-10). Globally, the people are always searching the eco-friendly alternative options to control the mosquitoes. For that, exploring of floral biodiversity is preferred, as they contain vast repository secondary metabolites. The tested plant, C. philippinum has larvicidal and pupicidal activity against An. stephensi which may be due to the presence of active biological compounds including terpenoids, flavonoid, alkaloids and phenolics etc. ${ }^{28-29}$ The above mentioned compounds may jointly or independently contribute to impact on larvicidal and pupicidal activity against An. stephensi and a similar report was given by Subarani et al. in which aqueous and solvent leaf extracts of Catharanthus roseus is able to impact on An. stephensi. ${ }^{4}$ The other effective results for malarial vector An. stephensi were also reported against different plant extracts such as Euphorbia hirta, ${ }^{11}$ Leucas aspera, ${ }^{30}$ Citrus sinensis, ${ }^{31}$ Gliricidia sepium. ${ }^{32}$ However, the ethanol extracts showed potential mortality in leaf and peel extract of Citrus sinensis and Gliricidia sepium, respectively as suggested from $\mathrm{LC}_{50}$ and $\mathrm{LC}_{90}$ values. ${ }^{31-32}$

The immature stages of mosquitoes are more susceptible to control as not only evidenced by An. stephensi but also from Ae. Aegypti against leaf extracts of C. philippinum (Tables 6-10). The synthetic toxins have been proved to be effective, but they pose some hazardous substances. Due to aquatic condition, they cause adverse effects on the environment and human health ${ }^{33}$ and hence, this finding not only helps to control the spreading of mosquito but also biodegradable and easily available in low cost. ${ }^{34}$ Plants possessing bioactive compounds are main the culprit against mortality of Ae. Aegypti which was suggested by Gandhi et al. after experimented on roots of Rubia cordifolia with $\mathrm{LC}_{50}$ and $\mathrm{LC}_{90}$ values of 3.86 and $8.28 \mathrm{ppm}$ for larvae, and 3.92 and $8.05 \mathrm{ppm}$ for pupae of A. aegypti. ${ }^{35}$ Further, these group isolated alizarin from roots of $R$. cordifolia, which is the main source to destroy the larva/ pupa of mosquito. Larvicidal and pupicidal actions of methanol leaf extract of Tephrosia purpurea was also observed against $A$. aegypti and found that the $\mathrm{LC}_{50}$ values of 1 st instar to $4^{\text {th }}$ larval instars were $139.24,176.24,219.28,256.27$, and $326.29 \mathrm{ppm}$, respectively whereas $\mathrm{LC}_{50}$ value of pupa was $326.29 \mathrm{ppm} .{ }^{15}$ The results so obtained also having similarities to Amir et al. who have taken the direct leaf and stem extracts of Parthenium hysterophorus against $A$. aegypti and confirmed as potential natural larvicidal agent. ${ }^{12}$

In our present study, the ethanol and methanol extracts of $C$. philippinum possessed higher larvicidal and pupicidal activities against An. stephensi and Ae. Aegypti if compare to aqueous, $\mathrm{n}$-hexane and chloroform. This variation was attributed with dissolving properties of the bioactive compounds in respect to solvent system or the polarity of the solvents. ${ }^{36}$ However, the mode of action of plant extracts on mosquito larvae are still unknown and up to some extent, it is postulated that the active phytochemicals interfering with the proper functioning of mitochondria more specifically at the proton transferring sites. ${ }^{37}$ Moreover, some secondary metabolites are inhibiting the growth of mosquito larvae by interacting with the midgut epithelium, gastric caeca and the malpighian tubules. ${ }^{38-40}$

\section{CONCLUSION}

The finding of the current investigation revealed that the leaf extracts of C. philippinum possess potential mosquito larvicidal and pupicidal activity against An. stephensi and Ae. aegypti. Since there is no availability of previous larvicidal and pupicidal activity of the selected plant species, this investigation serves as first-hand information. However, these results need corroboration further by characterizing, size estimating and determining the mode of action of individual bioactive compounds from leaf against both larval and pupal stages of development. In the future, this attempt may be recommended as an alternative tool to control the actions of An. stephensi and Ae. aegypti at their early life cycle processes.

\section{ACKNOWLEDGEMENT}

The authors are grateful to the Head, Post Graduate Department of Botany, Utkal University for providing necessary laboratory facilities.

\section{CONFLICT OF INTEREST}

The authors declare no conflict of interest.

\section{ABBREVIATIONS}

LC $_{50}$ : Lethal Concentration 50; LC $_{90}$ : Lethal Concentration 90; PPM: Parts Per Million.

\section{REFERENCES}

1. Reegan AD, Gandhi MR, Paulraj MG, et al. Ovicidal and oviposition deterrent activities of medicinal plan extracts against Aedes aegypti L. and Culex quinquefasciatus Say mosquitoes (Diptera: Culicidae). Osong Public Health Res Perspect. 2015;6(1):64-9.

2. Teklehaimanot A, Pushpa RJ. The mosquito: Public enemy No. 1. World Health Organization, Geneva. 1999.

3. Ghosh A, Chowdhury N, Chandra G. Plant extracts as potential larvicides. Indian J Med Res. 2012;135(5):581-98.

4. Subarani S, Sabhanayakam S, Kamaraj C, et al. Efficacy of larvicidal and pupicidal activity of Catharanthus roseus aqueous and solvent extracts against Anopheles stephensi Liston and Culex quinquefasciatus Say (Diptera: Culicidae). Asian Pac J Trop Med. 2013;6(8):625-30.

5. Panda S, Ranjit MR, Sahoo SL, et al. Larvicidal, pupicidal, adulticidal and repellent activity of Artemisia nilagirica (C.B.CI) Pamp against malarial vector Anopheles stephensi. Advan life Sci. 2016;5(11):4424-8.

6. Akram DS, Ahmed S. Dengue fever. Infect Dis J. 2005;14:124-5.

7. Dash AP, Valecha N, Anvikar AR, et al. Malaria in India: Challenges and opportunities. J Biosci. 2008;33(4):583-92.

8. Thomas S, Ravishankaran S, Justin NAJA, et al. Resting and feeding preferences of Anopheles stephensi in an urban setting, perennial for malaria. Malar J. 2017;16(11):1-7.

9. Cecilia D. Current status of dengue and chikungunya in India. WHO South East Asia J Public Health. 2014;3(1):22-7.

10. Porto KRDA, Motti PR, Yano M et al. Screening of plant extracts and fractions on Aedes aegypti larvae found in the state of MatoGrosso do Sul (Linnaeus, 1762) (culicidae). Ann Braz Acad Sci. 2017;89(2):895-906

11. Panneerselvam C, Murugan K, Kovendan K, et al. Mosquito larvicidal and pupicidal activity of Euphorbia hirta Linn. (Family: Euphorbiaceae) and Bacillus sphaericus against Anopheles stephensi Liston. (Diptera: Culicidae). Asian Pac J Trop Med. 2013;6(2):102-9

12. Amir $\mathrm{H}$, Butt $\mathrm{BH}$, Vehra SE. Evaluation of larvicidal activity of Parthenium hysterophorus against Aedes aegypti. Int J Mosq Res. 2017;4(2):1-4.

13. Panda S, Rout JR, Pati P, et al. Antimalarial activity of Artemisia nilagirica against Plasmodium falciparum. J Parasit Dis. 2017; https://doi.org/10.1007/s12639-0170956-9.

14. Govindarajan M, Sivakumar R, Rajeswari M, et al. Chemical composition and larvicidal activity of essential oil from Mentha spicata (Linn.) against three mosquito species. Parasitol Res. 2012;110(5):2023-32.

15. Venkadachalam $R$, Subramaniyan $V$, Palani $M$, et al. Mosquito larvicidal and pupicidal activity of Tephrosia purpurea Linn. (Family: Fabaceae) and Bacillus sphaericus against, dengue vector, Aedes aegypti. Pharmacogn J. 2017;9(6):737-42

16. Unnikrishnan G. Larvicidal and pupicidal activity of Terminalia catappa leaf extracts on Aedes aegypti mosquito: a vector intervention. IOSR J Pharm Biol Sci. 2014;9(2):58-63

17. Valentina J, Poonguzhali TV, Josmin LNLL. Mosquito larvicidal and pupicidal activity of seaweed extracts against Aedes aegypti, Anopheles stephensi and Culex quinquefasciatus. Int J Mosq Res. 2015;2(4):54-9.

18. Kar MK, Swain TM, Mishra SK. Antidiabetic activity of Clerodendrum philippinum Schauer leaves in streptozotocin induced diabetic rats. Int J Pharm Pharm Sci. 2015;7(9):386-9

19. Anonymous. Bloemen/ flowers: Clerodendrum philippinum-peanut butterplant Palma Verde Exoten 'Specialist in mediterrane planten en exoten'. Nederland 2017. (https://www.palmaverde.nl/en/clerodendrum-philippinum-peanutbutterplant.html).

20. Venkatanarasimman B, Rajeswari T, Padmapriya B. Preliminary phytochemical screening of crude leaf extract of Clerodendrum philippinum Schauer. Int J Instit Pharm Life Sci. 2012a;2(2):133-8.

21. DeSilva EDUD, Herath MNK, Perera PAGSK, et al. Genetic modification of rosa pinna (Clerodendrum philippinum Schauer) with gai mutant gene for improved plant architecture. Trop Agri Res. 2013;25(1):27-37. 
22. Borzabad RK, Sudarshana S, Hanumanthu M. In vitro plant regeneration from leaf explants of Artemisia vulgaris L. a medicinal herb. Mod Appl Sci. 2010;4(9):130-4

23. Shrivastava N, Patel T. Clerodendrum and heathcare: an overview. Med Aromat Plant Sci Biotechnol. 2007;1(1):209-23.

24. Venkatanarasimman B, Rajeswari T, Padmapriya B. Antibacterial potential of crude leaf extract of Clerodendrum philippinum Schauer. Int J Pharm Biol Arch. 2012b;3(2):307-10

25. Pushpavathi D, Shilpa M, Tejaswini $P$, et al. Evaluation of antifungal activity of some plants against seed-borne fungi. Sch J Agric Vet Sci. 2017;4(4):155-9.

26. World Health Organization. Guidelines for laboratory and field testing of mosquito larvicides. World Health Organization, Geneva. WHO/CDSMHOPES/ GCDPP/2005.13. 2005.

27. Finney DJ. Probit analysis. London: Cambridge University Press. 1971;68-78.

28. Udayan D, Nair SN, Padinchareveetil SK, et al. Evaluation of phytochemical constituents, proximate and fluorescence analysis of ethanolic extract and its fractions of Clerodendrum philippinum Schauer found in Wayanad region of Kerala, India. Res J Chem Sci. 2014;4(9):1-6.

29. Wang J, Luan F, He X, et al. Traditional uses and pharmacological properties of Clerodendrum phytochemicals. J Trad Comp Med. 2018;8:24-38.

30. Kovendan K. Studies on larvicidal and pupicidal activity of Leucas aspera Willd. (Lamiaceae) and bacterial insecticide, Bacillus sphaericus, against malarial vector, Anopheles stephensi Liston. (Diptera: Culicidae). Parasitol Res. 2012;110(1):195-203.

31. Murugan K, Mahesh KP, Kovendan K, et al. Larvicidal, pupicidal, repellent and adulticidal activity of Citrus sinensis orange peel extract against Anopheles stephensi, Aedes aegyptiand Culex quinquefasciatus (Diptera: Culicidae). Parasitol Res. 2012;111(4):1757-69.

32. Krishnappa K, Dhanasekaran S, Elumalai K. Larvicidal, ovicidal and pupicidal activities of Gliricidia sepium (Jacq.) (Leguminosae) against the malarial vector, Anopheles stephensi Liston (Culicidae: Diptera). Asian Pac J Trop Med. 2012 5(8):598-604.

33. Raveen R, Ahmed F, Pandeeswari M, et al. Laboratory evaluation of a few plant extracts for their ovicidal, larvicidal and pupicidal activity against medically important human dengue, chikungunya and zika virus vector, Aedes aegypti Linnaeus 1762 (Diptera: Culicidae). Int J Mosq Res. 2017;4(4):17-28.

34. Nasir S, Nasir I, Asrar M, et al. Larvicidal and pupicidal action of medicinal plant extracts against dengue mosquito Aedes albopictus (Skuse) (Diptera: Culicidae). Indian J Anim Res. 2017;51(1):155-8.

35. Gandhi MR, Reegan AD, Ganesan P, et al. Larvicidal and pupicidal activities of alizarin isolated from roots of Rubia cordifolia against Culex quinquefasciatus Say and Aedes aegypti (L.) (Diptera: Culicidae). Neotrop Entomol. 2016;45(4):441-8.

36. Nagappan, R. Evaluation of aqueous and ethanol extract of bioactive medicinal plant, Cassia didymobotrya (Fresenius) Irwin and Barneby against immature stages of filarial vector, Culex quinquefasciatus Say (Diptera: Culicidae). Asian Pac J Trop Biomed. 2012;2(9):707-11.

37. Usta J, Kreydiyyeh S, Bakajian K, et al. In vitro effect of eugenol and cinnamaldehyde on membrane potential and respiratory complexes in isolated rat liver mitochondria. Food Chem Toxicol. 2002;40(7):935-94.

38. Rey D, Pautou MP, Meyran JC. Histopathological effects of tannic acid on the midgut epithelium of some aquatic dipteral larvae. J Invert Pathol. 1999;73(2):173-81.

39. David JP, Rey D, Pautou MP, et al. Differential toxicity of leaf litter to dipteran larvae of mosquito developmental sites. J Invert Pathol. 2000;75(1):9-18

40. Panneerselvam C, Murugan K, Kovendan K, et al. Mosquito larvicidal, pupicidal, adulticidal, and repellent activity of Artemisia nilagirica (Family: Compositae) against Anopheles stephensi and Aedes aegypti. Parasitol Res. 2012;111(6): 2241-51.

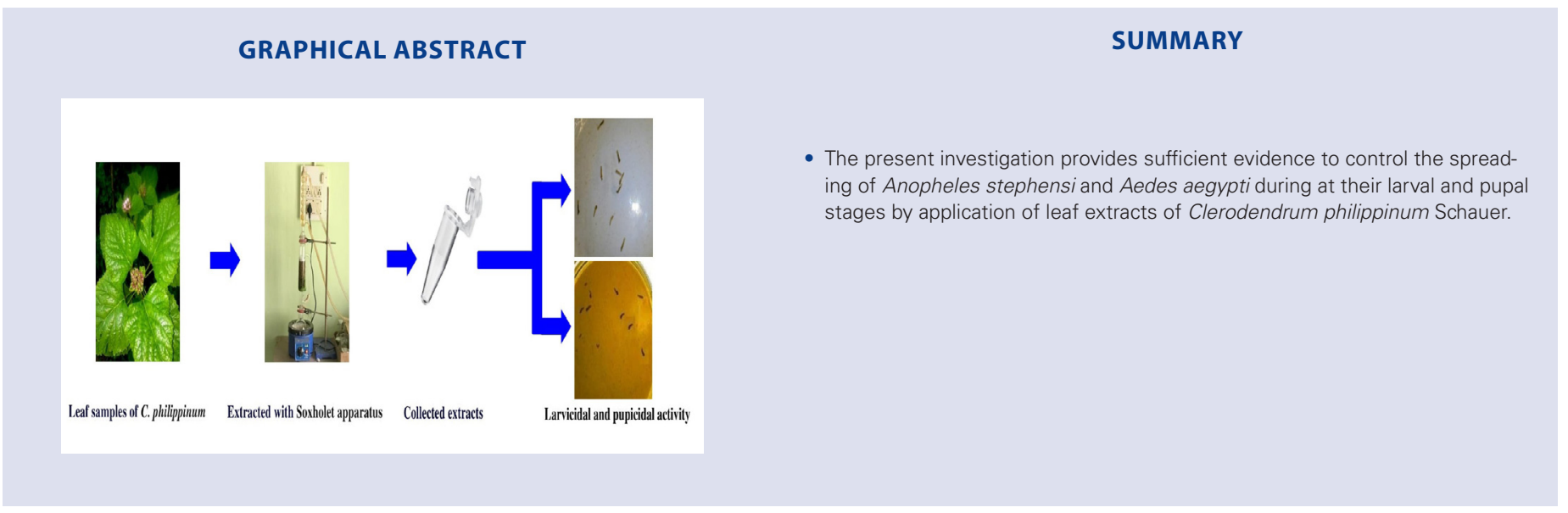

Cite this article: Dhal P, Rout JR, Dash PK, Panda S, Pati P, Rath CC, Pradhan C, Sahoo SL. Larvicidal and Pupicidal activity of Clerodendrum philippinum Schauer Leaf Extracts against Anopheles stephensi and Aedes aegypti. Pharmacog J. 2018;10(6):113742. 